\title{
XBotCore: A Real-Time Cross-Robot Software Platform
}

Conference Paper · April 2017

DOI: $10.1109 /$ IRC.2017.45

CITATIONS

0

6 authors, including:

\section{Luca Muratore}

Istituto Italiano di Tecnologia

5 PUBLICATIONS 16 CITATIONS

SEE PROFILE

Nikos G Tsagarakis

Istituto Italiano di Tecnologia

311 PUBLICATIONS $\quad \mathbf{4 , 7 3 0}$ CITATIONS

SEE PROFILE
READS

111
Alessio Rocchi

Istituto Italiano di Tecnologia

12 PUBLICATIONS 74 CITATIONS

SEE PROFILE

Some of the authors of this publication are also working on these related projects:

Project CENTAURO - Robust Mobility and Dexterous Manipulation in Disaster Response by Fullbody

Telepresence in a Centaur-like Robot View project

Project

WALK-MAN View project

All content following this page was uploaded by Luca Muratore on 30 April 2017.

The user has requested enhancement of the downloaded file. All in-text references underlined in blue are added to the original document and are linked to publications on ResearchGate, letting you access and read them immediately. 


\title{
XBotCore: A Real-Time Cross-Robot Software Platform
}

\author{
Luca Muratore $^{1}{ }^{2}$, Arturo Laurenzi ${ }^{1}$, Enrico Mingo Hoffman ${ }^{1}$, Alessio Rocchi ${ }^{1}$, \\ Darwin G. Caldwell ${ }^{1}$ and Nikos G. Tsagarakis ${ }^{1}$
}

\begin{abstract}
In this work we introduce XBotCore (CrossBot-Core), a light-weight, Real-Time (RT) software platform for EtherCAT-based robots. XBotCore is open-source and is designed to be both an RT robot control framework and a software middleware. It satisfies hard RT requirements, while ensuring $1 \mathrm{kHz}$ control loop even in complex Multi-DegreeOf-Freedom systems. It provides a simple and easy-to-use middleware Application Programming Interface (API), for both RT and non-RT control frameworks. This API is completely flexible with respect to the framework a user wants to utilize. Moreover it is possible to reuse the code written using XBotCore API with different robots (cross-robot feature). In this paper, the XBotCore design and architecture will be described and experimental results on the humanoid robot WALK-MAN [17], developed at the Istituto Italiano di Tecnologia (IIT), will be presented.
\end{abstract}

\section{INTRODUCTION}

One of the main challenges when developing a complex robotic system is the design and the implementation of a software architecture, essential for the interaction and the coordination of hardware and control modules. Ever more frequently a robot control system has to be able to perform critical tasks in an autonomous way, satisfying hard RT requirements, i.e. it must guarantee predictable response times. Furthermore it is necessary to have a software middleware capable of abstracting the complex hardware (e.g. actuators and sensors) of the robot providing a simple, standardized API to control the system. The robotics middleware should be modular, easy-to-use, robust, reliable, easy to maintain, efficient, flexible and should provide support for multithreading [4].

As a distributed control system the hardware components of the robot have to communicate using a field-bus system with RT communication capabilities: we selected EtherCAT (Ethernet Control Automation Technology), an industrial protocol built on the Ethernet (IEEE 802.3) specifications that assures:

- high transmission rate

- minimum roundtrip (reaction) time, w.r.t. other industrial protocols (e.g. CAN, Profibus, etc.) [13]

- precise synchronization $(\ll 1 \mu \mathrm{s})$ by exact adjustment of Distributed Clocks

- flexible topologies: Line, Star, Tree, Daisy Chain + Drop Lines can be used in any combination

- easy configuration and implementation

- cost effectiveness

EtherCAT combines an efficient and relatively high speed message transmission, with the predictability imposed by a master/slave medium access control policy. All the message

\footnotetext{
${ }^{1}$ Advanced Robotics Department (ADVR), Istituto Italiano di Tecnologia, Genova, Italy

${ }^{2}$ School of Electrical and Electronic Engineering, The University of Manchester, M13 9PL, UK

\{luca.muratore, arturo.laurenzi,

enrico.mingo, alessio.rocchi, darwin.caldwell,

nikolaos.tsagarakis\}@it.it
}

reception, data processing and frame retransmission operations are made "on the fly" by the slave nodes, without any extra delays. Special hardware components, embedded in the slave's Ethernet interface, are responsible for these operations.

RT scheduling is essential for precise robot control period, especially for high-frequency (e.g. $1 \mathrm{kHz}$ ): there are several operating systems or platforms which support RT operation, like Windows CE, INtime, RTLinux, RTAI, Xenomai, QNX, VXWorks. We selected a Linux based RTOS because we want to avoid a licensed product that does not give us the possibility to modify the source code depending on our system. Xenomai is our choice because its design considers extensibility, portability and maintainability as well as low latency [2], furthermore it has been already used successfully in many robotics hardware.

XBotCore is not specific to a single robot or to a class of robots: its implementation is flexible, generic and crossrobot. Furthermore it does not depend on any existing software platform, but it gives to the user the opportunity to easily integrate any RT or non-RT framework.

\section{RELATED WORK}

In [3] a low level control framework, called OROCOS (Open Robot Control Software), is introduced, which provides a set of components for RT control of robotic systems. It relies on the Common Object Request Broker (CORBA) architecture, that allows inter-process and cross-platform interoperability for distributed robot control. We decided not to depend on any Inter-Process-Communication (IPC) framework in order to avoid increasing the complexity of the software platform.

Very similar to OROCOS is OpenRT-M [1], developed in Japan from 2002 under NEDOs (New Energy and Industrial Technology Development Organization) Robot challenge program. It is based on CORBA, so similar considerations as for OROCOS can be made w.r.t. the software complexity; moreover part of OpenRT-M documentation is in Japanese.

YARP (Yet Another Robot Platform) [11] and ROS (Robot Operating System) [14] are popular component-based framework for IPC that do not guarantee RT execution among modules/nodes. It is essential for us to have a component responsible for the RT control of the robot, making these frameworks only viable as external (high-level) software frameworks.

PODO [8] is the framework used by KAIST in HUBO during the DRC (Darpa Robotics Challenge) Finals. Its control system has RT control capabilities and its interprocess communication facilities are based on POSIX IPC; moreover it uses a shared memory system called MPC to exchange data between processes in the same machine. This heterogeneous system has the potential to cause confusion as it is unclear which architectural style must be used to communicate with a specific component [7]. 
In [16] an RT architecture based on OpenJDK is introduced (used by IHMC during the DRC Finals). Nevertheless, to their own admission [9], none of the commercially available implementations of the Java Real Time Specification had the performance required to run their controller. Existing Real-time Java Support is insufficient.

Considering the above limitations, we started developing XBotCore from scratch, in order to have a reliable RT control framework without depending on complex IPC framework.

\section{Design GoAls}

The design of a software platform that lies at the foundations of a complex system, such as a robotic system, is the most crucial phase in the software development process. XBotCore was designed to be both an RT control system and an easy-to-use, flexible and reusable middleware for RT or non RT tasks.

XBotCore design goals are the following:

- Hard RT control system: it must perform computation within predictable timing constraints

- $1 \mathrm{kHz}$ control frequency: robotics applications may require high frequency control loops, e.g. RT Pattern Generator for Biped Walking or haptics applications

- Cross-Robot compatibility: it has to work with any kind of EtherCAT-based robot, without any code modification. It is crucial to be able to reuse the software platform with different robots, or different part of the same robot

- External Framework integration: it has to be possible to use XBotCore as a middleware for any kind of external software framework (RT or non RT)

- Plug-in Architecture: users and third parties should be able to develop their own modules. In a robotic system platform we need an highly expandable software structure

- Light-weight: we don't want too many dependencies on other libraries, it should be easy to install and set up. Moreover we expect to run XBotCore on embedded PCs with low performance requirements in terms of memory and CPU. We therefore need a small footprint and to avoid high CPU usage

- Simplicity: it must be simple. Complex systems may have unneeded and over-engineered features. For robotics application we need the full control over the software platform. KISS ("Keep It Simple, Stupid") principle is essential; simplicity is a key goal in XBotCore design and unnecessary complexity should be avoided

- Flexibility: XBotCore has to be easily modified or extended in order to be used in applications or environments other than those for which it was specifically designed

- Open-source: open-source software provides transparency in the software implementation since any developer can study and modify the code, eventually to the benefit of the robotics community. Moreover a flexible license is essential for the free distribution of XBotCore in other open-source projects

\section{XBOTCORE}

As shown in Figure 1 XBotCore consists of 5 main components: EtherCAT master, Plugin Handler, XBotCoreModel, RT and non RT middleware API and Communication Handlers.

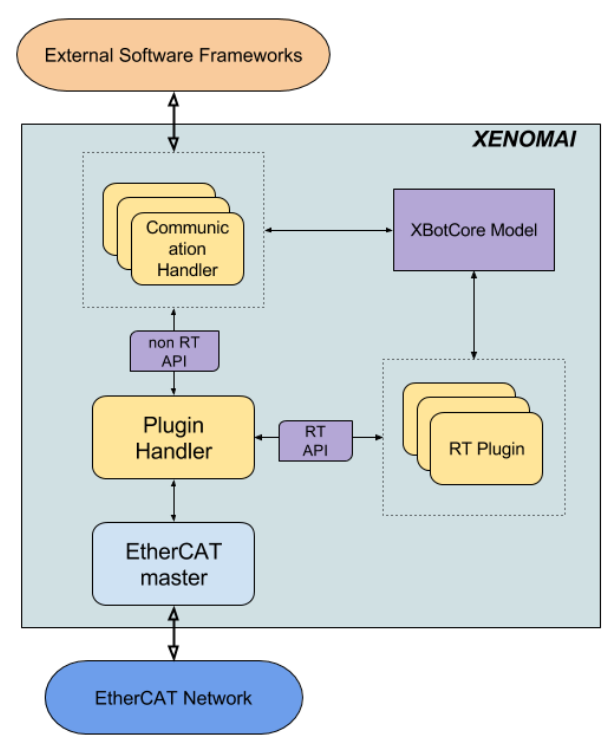

Figure 1. The diagram shows XBotCore components interactions: an EtherCAT master RT thread communicates with the EtherCAT slaves and with the Plugin handler RT thread which schedules execution of a set of RT plugins. The Communication Handlers non-RT threads allow interfacing with non-RT external Software Frameworks

\section{A. EtherCAT master}

XBotCore is designed for EtherCAT based robots: we expect a network of EtherCAT slaves in the system, e.g. the electronic boards responsible for motor control and sensors data acquisition. The EtherCAT protocol has a master/slave medium access control policy: XBotCore EtherCAT master implementation is developed starting from the SOEM (Simple Open EtherCAT Master) library, an open source implementation, meant to be highly portable on a variety of embedded platforms (HW and RTOSes) [12]. The structure of the data flowing in the EtherCAT network is called PDO (Process Data Object) and it has two different sub-structures: $P D O R X$ : master input, slave output e.g. link position, motor position, motor velocity, torque, temperature etc. and $P D O$ $T X$ : master output, slave input e.g. position reference, torque reference, gains etc.

Furthermore the XBotCore EtherCAT master provides an asynchronous API to the higher level components in order to $\mathrm{read} / \mathrm{write}$ the PDO data.

\section{B. Plugin Handler}

The Plugin Handler is the main component of the RT plugin architecture: it is an RT thread responsible to start all the loaded plugins, execute them sequentially (as in [19]), and close them before unloading them. A Plugin is a class inheriting from the abstract class XBotPlugin. The Plugin implementation is compiled as a shared object (.so). It is possible to dynamically load and unload one or more plugins in the Plugin Handler. Writing a Plugin is straightforward for the user, as he just needs to implement three functions:

- an init () function that will be called only once by the Plugin Handler in order to initialize the variables of the Plugin

- a run () function which will be executed in the control loop of the Plugin Handler 
- a close () function, called when the Plugin Handler wants to remove the plugin

\section{XBotCoreModel}

XBotCore implies a novel approach to the configuration of low-level control systems by using modern description formats such URDF (Universal Robotics Description Format) and SRDF (Semantic Robotic Description Format), traditionally used for high-level software components (e.g. ROS nodes). Its main feature is to be a cross-robot software platform: thanks to the abstractions provided by the XBotCoreModel class it is possible to control different robots or different parts of the same robot without code modifications. In fact the API provided to control the robot is dynamically built starting from the URDF and SRDF of the robot. Modifying the SRDF, removing for example a kinematic chain (e.g. the torso of the robot), results in a different API for the user that is compatible with the available/desired parts of the robot to control. The same happens when the URDF is modified, e.g. when working with a different robot.

\section{D. $R T$ and non-RT middleware API}

$X B o t$ Core is also a middleware that provides the user with both RT and non-RT APIs The RT API is suitable for the RT plugins that will run in the Plugin Handler: it works using a shared memory communication mechanism with the low level RT EtherCAT thread. The interfaces implemented by the RT API are: IXBot Joint (abstraction of the robot joints), IXBotchain (abstraction of the robot kinematic chain), IXBotRobot (abstraction of the robot) and IXBotFT (abstraction of the robot Force/Torque (F/T) sensors).

The non-RT API implements similar interfaces (i.e. IXBotJoint and IXBotFT, but it uses XDDP (Cross Domain Datagram Protocol) Xenomai pipes in order to have asynchronous communication between RT and non-RT threads. It is crucial to have a lock-free IPC in a robotic system: RT control threads are able to exchange messages with non-RT communication threads without any context switch.

\section{E. Communication Handlers}

A robotic system has to communicate with the external world using a set of non-RT threads: in XBotCore the XBotCommunicationHandler class is provided; instances of classes inheriting from XBotCommunicationHandler run in non-RT threads, from which developers have access to ready-to-use non-RT API functions.

It is pretty straightforward to implement a new set of Communication Handlers: XBotCore provides built-in support for YARP and ROS communication frameworks.

\section{EXPERIMENTS}

\section{A. Experiments description}

To validate and evaluate the performance of the XBotCore software platform, we performed a set of experiments on the WALK-MAN robot, a full-size humanoid with 33 DOFs (Degree-Of-Freedoms) and 4 custom F/T sensors. The WALK-MAN head is equipped with a CMU Multisense-SL sensor that includes a stereo camera, a 2D rotating laser scanner, and an IMU. The robot control modules are based on GYM [5] (Generic Yarp Module), a component model

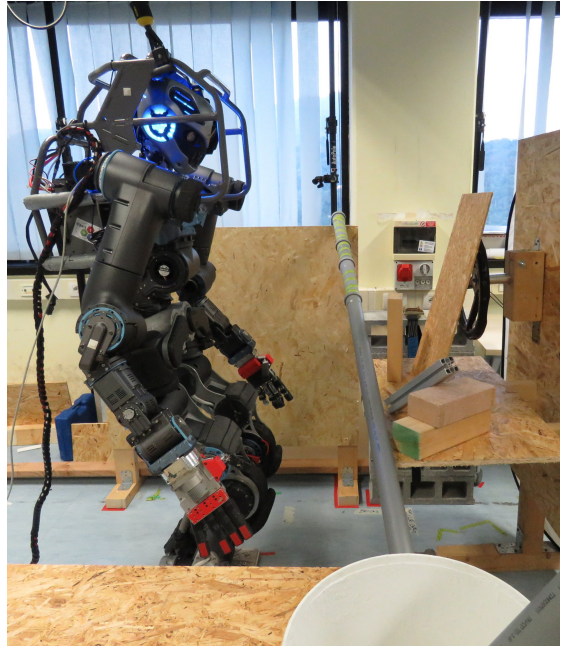

Figure 2. XBotCore validation experiments setup: WALKMAN needs to remove a set of objects in order to perform the valve turning

to easily develop software modules for robotics leveraging the YARP ecosystem: Yarp Based Plugins for Gazebo Simulator [6] were used to validate the control modules in simulation. Whole-body control and inverse kinematics are solved through the OpenSoT control framework [15].

In the evaluation different high-level software frameworks were successfully integrated on top of XBotCore: ArmarX [18] perceptual pipeline for hierarchical affordance extraction [10], Open-SoT previewer based on the MoveIt! ROS library for motion feasibility analysis and collision checking and a manipulation GYM module, Open-SoT based, using the YARP communication framework.

The experiments were carried out in a DRC-inspired scenario targeting the removal of debris in front of a valve. In Figure 2) the experimental setup is shown.

\section{B. Results}

We analyzed XBotCore performance in terms of control period of the RT plugins and CPU usage: during the experiments, each millisecond, we recorded all the data flowing from the EtherCAT master to the EtherCAT slaves and vice versa, thanks to an XBotCore low-level logging tool.

In Figure 3) we show the control period measured during the experiments in the worst-case scenario, i.e. while the robot was performing the manipulation actions: it is clear that the control period is always below the $1000 \mu$ s (i.e. $1 \mathrm{kHz}$ control frequency) even if the RT system is communicating with the high-level software components through YARP XBotCommunicationHandler non-RT threads.

In Figure 4) a comparison is presented between XBotCore CPU usage while the robot is idle (i.e. not moving, nor communicating with external software frameworks) and when the manipulation experiments are running: the CPU core usage overhead introduced by XBotCore when the robot is performing the manipulation task as described above, is only $1.2 \%$ (in average). Furthermore it is clear that the CPU usage of XBotCore is very low (always ranging from $11.7 \%$ to $14.2 \%$ ). 


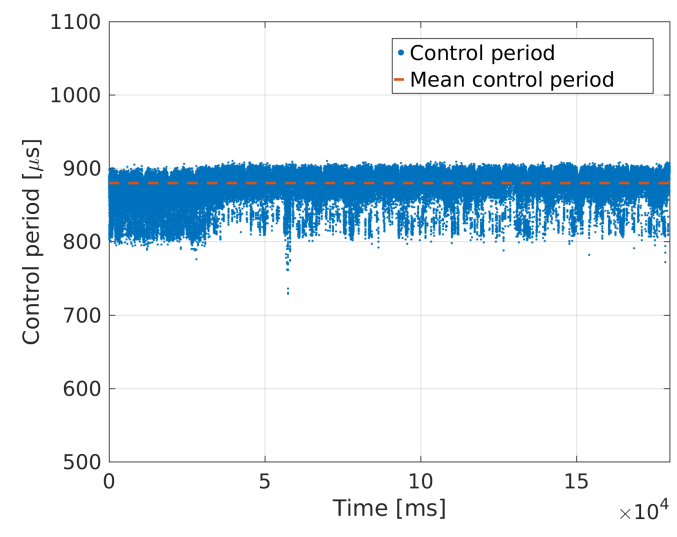

Figure 3. WALK-MAN control period during manipulation actions: XBotCore assures always a control period below $1000 \mu \mathrm{s}$

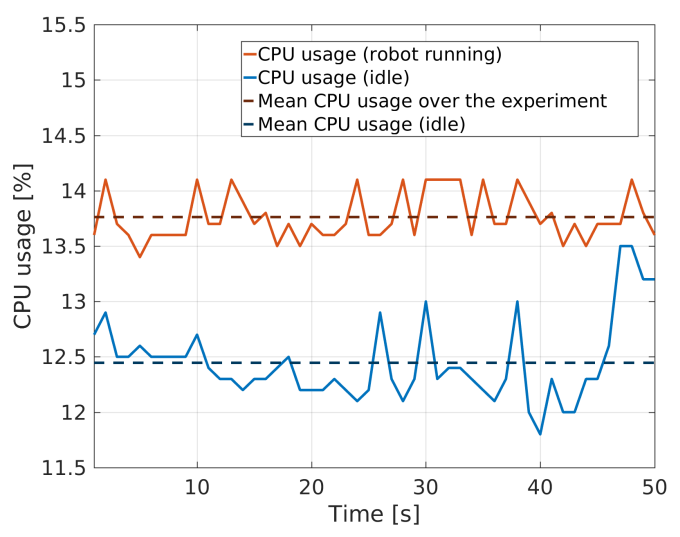

Figure 4. XBotCore CPU core usage comparison: robot idle vs robot running the experiments

\section{CONCLUSIONS}

In this work we introduced an RT, robot-agnostic software platform called XBotCore. XBotCore works with any EtherCAT based robot and it has a plugin-based software architecture: it is easy and not time-consuming to write a new RT control plugin. As a final remark we showed that external frameworks (e.g. ROS or YARP) are easy to integrate in XBotCore, thanks to his middleware API. We evaluated XBotCore performance on the humanoid robot WALK-MAN, showing that it can ensure $1 \mathrm{kHz}$ RT control loop during complex manipulation tasks, while communicating with the non RT external frameworks. Moreover we demonstrated that XBotCore is light-weight: the CPU usage is very low, both when the robot is idle and when it is executing a task. XBotCore is released free and open source at https://gitlab.robotology.eu/luca. muratore/xbotcore

\section{ACKNOWLEDGMENTS}

The research leading to these results has received funding from the European Union Seventh Framework Programme [FP7-ICT-2013-10] under grant agreements n.611832 WALKMAN, and European Unions Horizon 2020 research and innovation programme under grant agreement
No 644839 (CENTAURO) and 644727 (CogIMon). The authors would like to thank Lorenzo Natale, Ali Paikan, Alberto Cardellino and Alessio Margan for the support. The authors want also to thank Tamim Asfour, Peter Kaiser and Markus Grötz from High Performance Humanoid Technologies Lab $(\mathrm{H} 2 \mathrm{~T})$ in KIT, for their collaboration in the preparation of the experimental setup.

\section{REFERENCES}

[1] N. Ando, T. Suehiro, K. Kitagaki, T. Kotoku, and W.-K. Yoon. Rtmiddleware: distributed component middleware for rt (robot technology). In 2005 IEEE/RSJ International Conference on Intelligent Robots and Systems, pages 3933-3938. IEEE, 2005.

[2] J. H. Brown. How fast is fast enough? choosing between xenomai and linux for real-time applications. Twelfth Real-Time Linux Workshop, 2012.

[3] H. Bruyninckx. OROCOS: design and implementation of a robot control software framework. Proc. IEEE RAS EMBS Int. Conf. Biomed. Robot. Biomechatron., 2002.

[4] A. Elkady and T. Sobh. Robotics middleware: A comprehensive literature survey and Attribute-Based bibliography. Journal of Robotics, 2012, 7 May 2012.

[5] M. Ferrati, A. Settimi, L. Muratore, N. G. Tsagarakis, L. Natale, and L. Pallottino. The Walk-Man robot software architecture. Front. Robot. AI, 3, 12 Apr. 2016.

[6] E. M. Hoffman, S. Traversaro, A. Rocchi, M. Ferrati, A. Settimi, F. Romano, L. Natale, A. Bicchi, F. Nori, and N. G. Tsagarakis. Yarp based plugins for gazebo simulator. In International Workshop on Modelling and Simulation for Autonomous Systems, pages 333-346. Springer International Publishing, 2014.

[7] T. Houliston, J. Fountain, Y. Lin, A. Mendes, and others. NUClear: A loosely coupled software architecture for humanoid robot systems. Frontiers in Robotics, 2016.

[8] L. Jeongsoo, L. Jungho, and O. Jun-Ho. Development of robot software framework podo: Toward multi-processes and multi-users. Workshop on software architectures and methodologies for developing humanoid robots, IEEE HUMANOIDS 2014, 2014.

[9] M. Johnson, B. Shrewsbury, S. Bertrand, T. Wu, D. Duran, M. Floyd, P. Abeles, D. Stephen, N. Mertins, A. Lesman, J. Carff, W. Rifenburgh, P. Kaveti, W. Straatman, J. Smith, M. Griffioen, B. Layton, T. de Boer, T. Koolen, P. Neuhaus, and J. Pratt. Team IHMC's lessons learned from the DARPA robotics challenge trials. J. Field Robotics, 32(2):192-208, 1 Mar. 2015.

[10] P. Kaiser, M. Grotz, E. E. Aksoy, D. Kanoulas, N. G. Tsagarakis, and T. Asfour. Experimental evaluation of a perceptual pipeline for hierarchical a ordance extraction. International Symposium on Experimental Robotics ISER, 2016.

[11] G. Metta, P. Fitzpatrick, and L. Natale. Yarp: Yet another robot platform. International Journal on Advanced Robotics Systems, 2006.

[12] D. Orfanus, R. Indergaard, G. Prytz, and T. Wien. EtherCAT-based platform for distributed control in high-performance industrial applications. In 2013 IEEE 18th Conference on Emerging Technologies Factory Automation (ETFA), pages 1-8. ieeexplore.ieee.org, 2013.

[13] S. Potra and G. S. LVD-Napomar. EtherCAT protocol implementation issues on an embedded linux platform. Automation, Quality and Testing, Robotics, 2006 IEEE International Conference on, 2006.

[14] M. Quigley, K. Conley, B. P. Gerkey, J. Faust, T. Foote, J. Leibs, R. Wheeler, and A. Y. Ng. Ros: an open-source robot operating system. In ICRA Workshop on Open Source Software, 2009.

[15] A. Rocchi, E. M. Hoffman, D. G. Caldwell, and N. G. Tsagarakis. Opensot: a whole-body control library for the compliant humanoid robot coman. In 2015 IEEE International Conference on Robotics and Automation (ICRA), pages 6248-6253, 2015.

[16] J. Smith, D. Stephen, A. Lesman, and J. Pratt. Real-time control of humanoid robots using openjdk. In Proceedings of the 12th International Workshop on Java Technologies for Real-time and Embedded Systems, JTRES '14, pages 29:29-29:36, New York, NY, USA, 2014. ACM.

[17] N. G. Tsagarakis, D. G. Caldwell, A. Bicchi, F. Negrello, M. Garabini, W. Choi, L. Baccelliere, V. Loc, J. Noorden, M. Catalano, M. Ferrati, L. Muratore, A. Margan, L. Natale, E. Mingo, H. Dallali, J. Malzahn, A. Settimi, A. Rocchi, V. Varricchio, L. Pallottino, C. Pavan, A. Ajoudani, J. Lee, P. Kryczka, and D. Kanoulas. WALK-MAN: A High Performance Humanoid Platform for Realistic Environments. Journal of Field Robotics (JFR), 2016.

[18] N. Vahrenkamp, M. Wächter, M. Kröhnert, K. Welke, and T. Asfour The robot software framework ArmarX. it - Information Technology, 57(2), 2015.

[19] K. Yokoi, F. Kanehiro, K. Kaneko, S. Kajita, K. Fujiwara, and H. Hirukawa. Experimental study of humanoid robot HRP-1S. Int. J. Rob. Res., 23(4-5):351-362, 1 Apr. 2004 